\title{
Finite element predictions of residual stresses due to heat transfer during welding
}

\author{
S. Syngellakis ${ }^{1} \& \mathrm{~A} . \mathrm{Wu}^{2}$ \\ ${ }^{1}$ Wessex Institute of Technology, UK \\ ${ }^{2}$ Tongji University, China
}

\begin{abstract}
Finite element simulations of a single pass butt and a sequential fillet welding process are critically reviewed. Temperature history as well as welding-induced distortion and residual stress data from tests were compared to the results from the simulations for calibration and validation purposes. The main issue addressed was the sensitivity of the temperature and residual stress predictions to variations of heat input, temperature-dependent material properties as well as initial and boundary conditions. Key modelling features included an essentially 2D analysis combined with a ramped heat input function; modelling of radiation and fit-up; accounting for plastic properties variations in the welding area; the use of element death and re-birth technique to simulate sequential welding.

Keywords: heat transfer, residual stresses, finite elements, butt weld, fillet weld, plasticity, temperature-dependent properties.
\end{abstract}

\section{Introduction}

The prediction of welding-induced residual stresses and distortion is essential to the reliable design of welded joints; due however to the complexity of the welding process and the large number of modelling parameters involved, it often relies on simplifying assumptions and empirical relations. The experimental measurement of residual stresses has provided useful insight into their possible distribution and magnitude but the scope of such methods is necessarily limited due to their cost and potential error sources; most of them are also destructive and provide stress information at discrete points. A reliable and efficient numerical simulation of the welding process is thus considered as a more desirable alternative. This has already been attempted by many investigators; in- 
house or commercial, general-purpose finite element (FE) codes, such as ANSYS, have been used for that purpose.

Important modelling considerations in FE simulations of the welding process have been the heat source [1, 2]; radiation [3], phase transformation [4] and transformation plasticity [5]; the effect of moving arc in two dimensional models [6]; multi-pass welding; the interaction between the weld zone and the overall elastic structure [7,8]. Special modelling techniques were applied, such as combined surface and body flux components to simulate arc heat effects [2], element rebirth to simulate the deposition of weld metal [6]; re-zoning and substructuring to improve modelling efficiency [3].

FE analyses need to be validated by comparing their predictions with available experimental and other numerical data. They can also be simplified by examining the sensitivity of their output to variations of input parameters. Such a sensitivity analysis is also necessitated by the inherent uncertainty associated with most of the material and loading input.

The possibility of generating robust and reliable FE models, which are relatively simple but also preserve all the essential welding features, is examined here through the review of recent simulations of butt [9] and fillet welding [10]. The modelling of butt-welds was based on published geometric, heat input and material data and its validation relied on comparisons with available residual stress measurements and distortion predictions from empirical formulae. A manufactured, sequentially fillet-welded $\mathrm{T}$-joint provided temperature and distortion measurements for comparison with the respective FE predictions for calibration and validation purposes. Since the variation of thermal and mechanical properties with temperature was not readily available, such information was retrieved from the relevant literature. The sensitivity of residual stress predictions to variations of the various input parameters and their temperature-dependence was investigated.

\section{Modelling considerations}

The temperature field is dominated entirely by the heat input from the welding heat source, thus the thermo-mechanical coupling has a negligible effect on the thermal analysis [11], which is conducted first to yield the temperature development due to the sudden transfer of heat from the welding arc and the gradual heat loss through boundary conduction, convection and radiation. A stress analysis is then performed with the already obtained temperature histories as loading input to yield the induced residual stresses. Both analyses were performed using ANSYS which allows their sequential application using an appropriate combination of elements. A four-node thermal solid element, used for both weld metal and base metal, was replaced by a generalised plane strain element for the elasto-plastic stress analysis. Thus, the same element mesh was used for both analyses.

The amount of heat input $Q$ supplied to the workpiece is obtained from

$$
Q=\eta V I
$$


where $V$ the arc voltage, $I$ the arc current and $\eta$ is the arc efficiency accounting for the direct heat loss from the arc to the surrounding environment through convection and radiation; $\eta$ has been empirically found to vary by up to $20 \%$ for various types of welding process. An amount of heat $Q_{m}$ is consumed to melting the electrode so that the net heat input is $Q_{a}=Q-Q_{m}$. Alternatively, $Q_{a}$ can be calculated through a melting efficiency $\eta_{m}$ defined as the ratio of the energy used for melting to that delivered to the workpiece [12]. The heat input $Q_{a}$ is assigned to the weld metal as surface load $Q_{s}$ and as body load $Q_{b}$. The choice of the ratio $Q_{s} / Q_{b}$ can be linked to achieving an accurate representation of the actual fusion zone by the FE model [2]. A uniform $Q_{s}$ distribution is usually adopted since other choices have led to very similar final results. The body heat $Q_{b}$ is also applied uniformly to the weld metal.

Except within the regions near the ends of the welded plates, the majority of cross-sections have been observed to undergo the same thermal and mechanical history during welding; thus, a two-dimensional model of any cross-section perpendicular to the direction of the welding should be sufficient for predicting the behaviour of the whole joint. In such a $2 \mathrm{D}$ simulation of the $3 \mathrm{D}$ welding process, the analysed cross-section is in the middle region of the weldment, transverse to the welding direction. When the arc is at a certain distance ahead of that cross section or when it leaves the section, the base metal of the section obtains heat from the part under the arc through conduction. A ramped heat input function, as shown in fig. 1, has been adopted to include the effect of out-ofplane heat input as well as to avoid numerical divergence problems, caused by a step temperature change. Since a solid of unit thickness is analysed, the welding time $t_{2}$ is equal to $1 / v_{w}$, where $v_{w}$ is the welding speed; the ratio $t_{1} / t_{2}$ can be taken equal to 0.2 , found to give best correlation with experimental data $[2,6]$.

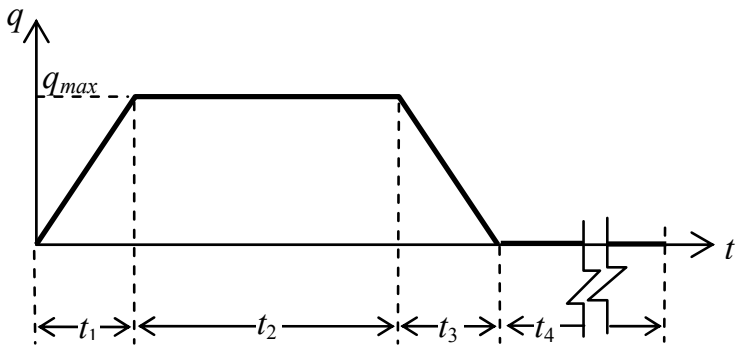

Figure 1: Ramped heat input function.

Since the weld metal is deposited into the weldment in a molten state, its melting point $T_{m}$ is assigned as its initial temperature combined with $Q_{a}$ as the net heat input. The weld metal can also be assumed initially at room temperature $T_{r}$ but then $Q$, calculated from eqn (1), should be the heat input. The base metal is heated up from $T_{r}$, which is assigned as its initial temperature. 
An extensive literature search for property data indicated the extent of their variability for different types of steel at various temperature levels. The thermal properties used by a group of researchers [13-15] showed considerable consistency although collected from different sources. With regard to specific heat $c(T)$, a particular set of data [16] was tested and found reliable by other investigators $[4,5]$. Other $c(T)$ curves $[17,18]$ were very different from the rest but their origin was not mentioned although the distinctive character of certain data may be attributed to the material being stainless rather than mild steel [18]. Significant deviations in the temperature dependence of specific heat data were partly due to the omission of the latent heat associated with phase transformations. Enthalpy $H(T)$ rather than $c(T)$ is the input required by ANSYS in the case of transient problems involving phase change. The versions of $H(T)$, obtained from various $c(T)$ data retrieved from the relevant literature, were remarkably consistent.

All information on temperature dependence of mechanical properties in published welding simulations had been provided by manufacturers or retrieved from reference publications. The collected relevant data on the Young's modulus $E$ indicated that $E\left(T_{r}\right)$ varies little with the composition of structural steel; its rate of decrease is slow for temperatures up to $600 \mathrm{~K}$; then it drops to almost zero value at a rate depending on the melting temperature of the grade of steel used.

For comparison purposes, the reviewed variations of yield stress $\sigma_{Y}$ with $T$ were normalised with respect to $\sigma_{Y}\left(T_{r}\right)$. Most normalised curves showed three distinct rates of $\sigma_{Y}$ decrease with $T$. It is possible to account for the effect of the temperature history cycle on $\sigma_{Y}$ by using different curves for heating and cooling. Another issue, not addressed in previous FE work, is the difference between $\sigma_{Y}$ for the weld and base metals. Isotropic strain hardening has been most commonly assumed but since the material in and near the fusion zone experiences a cycle of compressive and then tensile plastic strain, the use kinematic strain hardening seems more rational.

Thermo-mechanical coupling can be modelled either through the thermal dilatation $\varepsilon_{T}$ or the thermal expansion coefficient $\alpha$. Values of the latter reported in the literature $[2,7,14]$ do not normally account for the volume change due to phase change. This effect is included in the reported values of $\varepsilon_{T}$, which, in certain cases, also depended of the whole temperature cycle, that is, different curves were used for heating and cooling $[13,15,16]$. The variation of thermal dilatation with temperature for steels of 0.26 and 0.49 carbon content showed that chemical composition affects only slightly the magnitude of $\varepsilon_{T}$ [19]. The mean thermal expansion coefficient, which can be obtained either from $\varepsilon_{T}(T)$ or $\alpha(T)$, was entered into the ANSYS program.

The free convection coefficient $h$ generally varies both spatially and with temperature. Minor variations in alloy content for structural steels were deemed to have negligible influence on $h$ [14]. Its effect on temperature and residual stress predictions has not be found significant; analyses were performed with a constant value of around $12 \mathrm{~W} / \mathrm{m}^{2} \mathrm{~K}$. 
The heat loss rate due to radiation $q_{r}$ can be calculated using [7]

$$
q_{r}=e \sigma\left(T^{4}-T_{r}^{4}\right)
$$

where $e$ is the emissivity and $\sigma=5.67 \times 10^{-8} \mathrm{~W} / \mathrm{m}^{2} \mathrm{~K}^{4}$ the Stefan-Boltzman constant. Radiation was neglected by several authors $[2,4,5,17]$ while others [14-16] simply adopted constant $e$ values ranging from 0.2 to 0.5 . Only Brown and Song [7] provided data for a temperature-dependent $e$. Although radiation can be independently modelled in ANSYS, it is also possible to combine it with convection by deriving an equivalent convection coefficient [13].

\section{Butt weld}

The weldment was assumed to be perfectly symmetric with respect to a longitudinal section through the centre of the weld so that only one half of it was modelled; no penetration or overfill was considered. The adopted values of $h(T)$ resulted from a detailed assessment of heat convection over the various boundaries of a welded joint at various temperatures [3]. The temperature history obtained from the thermal analysis was reviewed and ranges of high gradient were identified so that a high density of time steps could be defined only within these ranges. This increased the efficiency of the residual stress analysis.

Modelling was applied to a tested specimen with published experimental results so that the relevance of the FE predictions could be assessed. Rao and Tall [20] provided a significant amount of experimental evidence, consistent with that from other investigators, as well as additional background information for building a reasonably representative FE model. Among the various specimens tested, a single pass welding (labelled T-2-6) was selected for simplicity of modelling. A sketch of the left part of its cross-section is shown in fig. 2. Some dimensions were assumed based on common welding practice.

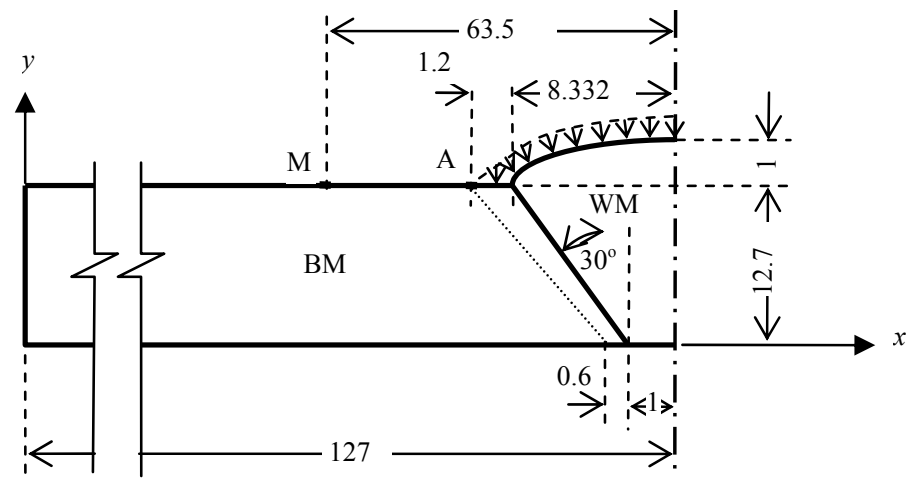

Figure 2: Butt-weld cross-section (dimensions in mm).

The surface heat $Q_{s}$ was applied as far as point A on the base metal surface, as shown in fig. 2. Convection and radiation was applied to all free surfaces, that is, 
on the top surface, to the left of point A, and on the left side at $x=0$. These conditions were extended to the right of point A after the surface heat flux was removed, that is, during time $t_{4}$ as defined in fig. 1 . The initial temperature of the weld metal was assumed to be equal to the melting temperature $T_{m}$.

A significant number of tensile tests on coupons extracted from welded specimens provided $\sigma_{Y}$ as well as elongation data for both base and weld metals at room temperature. These data were supplemented by temperature dependence information from the reviewed literature. The bottom surface of the weldment was reported to rest on a steel column block during welding [20]. Therefore, a significant heat conduction loss through that surface should have been expected. The support was assumed to be a continuous steel plate of thickness $\ell$ whose heat transfer effect was modelled as convection characterised by an equivalent coefficient $h_{e}$.

The initial thermal FE analysis relied on literature data for $V, I$ and $v_{w}$ [20], conductivity $k$ [14] and specific heat $c$ [16]; the latter was used to calculate $H(T)$. The implied shielded metal-arc welding led to the choice of the middlerange $\eta$ value of 0.75 . The values $293 \mathrm{~K}$ and $1803 \mathrm{~K}$ were adopted for $T_{r}$ and $T_{m}$, respectively. For the supported bottom surface, an average $h_{e}\left(T_{r}\right)$ of about 500 $\mathrm{W} / \mathrm{m}^{2} \mathrm{~K}$ was estimated based on an extreme heating and cooling cycle $T_{c}$ of approximately $300 \mathrm{~s}$, combined with typical values of $\rho, c$ and $k$ for steel at room temperature and a support plate thickness $\ell=45 \mathrm{~mm}$. The temperature dependence of $h_{e}$ was assumed to be given by

$$
h_{e}(T)=h_{e}\left(T_{r}\right) k(T) / k\left(T_{r}\right) .
$$

The analysis was also run with the value of $\eta$ changed to 0.66 and 0.85 , that is, its extreme values for metal-arc welding [21, 22]; the obtained temperature results indicated that the variation of the amount of heat input within its known empirical range has a significant effect on temperature development in the weld area. Using conductivity data [16] differing by a maximum of $27.5 \%$ at $T_{r}$ from those initially adopted resulted in an increase of the maximum temperature at A (fig. 2) by about $10 \%$. Accounting for radiation using a published record of $e(T)$ [7] increased the cooling rate only slightly. The analysis was also performed with a quite different $h(T)$ [17] from that initially chosen but no noticeable difference in the temperature histories was found. Due to the high degree of uncertainty in modelling the heat loss through the support of the bottom surface, the analysis was repeated with a much lower $h_{e}\left(T_{r}\right)=260 \mathrm{~W} / \mathrm{m}^{2} \mathrm{~K}$. This affected the cooling rate and a more significant difference in temperatures was seen further away from the weld area.

The adopted $E(T)$ was taken from Andersson [16], who is generally viewed as a reliable source. The reported average $\sigma_{Y}\left(T_{r}\right)$ values for base and weld metal were equal to $244 \mathrm{MPa}$ and $342.4 \mathrm{MPa}$, respectively [20]. Values of $450 \mathrm{MPa}$ and $440 \mathrm{MPa}$, corresponding to a strains of $15 \%$ and $12 \%$, were adopted for the ultimate tensile strength $\sigma_{u}\left(T_{r}\right)$ of the base and weld metal, respectively; This information was retrieved from reference publications. The normalised curves from Andersson [16] and Jonsson et al. [4] were used as a basis for generating $\sigma_{Y}(T)$ of the base and weld metals; $\sigma_{u}(T)$ was assumed to be similar to $\sigma_{Y}(T)$, 
accounting for the effect of a decreasing hardening modulus. Finally, the $0.26 \mathrm{C}$ $\varepsilon_{T}(T)$ was adopted, since the tested material [20] was a structural steel with normally less than $0.26 \%$ carbon.

With the initially assumed input, the extreme tensile and compressive stresses were found lower than the corresponding experimental measurements. Uncertainties regarding the heat input, the material properties and the assumed boundary conditions could affect the accuracy of the residual stress predictions. It was therefore important to examine the sensitivity of these results to rational variations of the initial input.

The effect of heat flux through interfaces with support elements, which was found to be strong on temperature history, was also assessed on residual stresses. The $\sigma_{z z}$ distribution along the top surface was obtained for $h_{e}\left(T_{r}\right)=260 \mathrm{~W} / \mathrm{m}^{2} \mathrm{~K}$ and $h_{e}(T)=h(T)$, that is, the adopted free convection coefficient. Comparisons with the solution obtained using the initially chosen input indicated that reducing $h_{e}$ by almost $50 \%$ has a moderate effect on the $\sigma_{z z}$ distribution but assuming free convection on the bottom surface leads to completely erroneous results. As with temperature histories, variations in heat input, conductivity and emissivity were found to have a smaller impact than those of $h_{e}$ on the residual stress predictions. Variations of heat input, in particular, did not affect residual stress predictions significantly despite their strong effect on temperature development in the weld area.

Increasing the $\sigma_{Y}\left(T_{r}\right)$ of both base and weld metal by $30 \%$ had the most significant impact on the magnitude of tensile and compressive longitudinal residual stress among all parameters studied previously. The effect of adopting different $\sigma_{Y}(T)$ keeping $\sigma_{Y}\left(T_{r}\right)$ constant was also examined but found to be minimal. Based on reported ranges of $\sigma_{u}\left(T_{r}\right)$ for the grades of steel used, the base and weld metal values of $\sigma_{u}\left(T_{r}\right)$ were increased by $20 \%$ relative to the initially assumed input while keeping $\sigma_{Y}\left(T_{r}\right)$ unchanged. The new prediction of the maximum tensile residual stress in the weld area was found to be higher by $12 \%$ than the original solution.

Based on the simulated test description [20], an approximate representation of the actual support arrangement was introduced into the model. The mechanical properties of the weld metal (WM) were modified at this stage to account for the fact that the WM tensile test pieces contained some proportion of base metal (BM) [20]. In view of the published ranges for $\sigma_{Y}$ and $\sigma_{u}$ for the electrode used, these two properties of WM were raised to $420 \mathrm{MPa}$ and $500 \mathrm{MPa}$, respectively. The resulting residual $\sigma_{z z}$ distribution along the top surface, for both the unconstrained and the constrained model, is shown in fig. 3. The FE analysis with the modified WM plastic properties is seen to give predictions in quite good agreement with the experimental results apart from the compressive residual stress towards the ends of the plate, which are underestimated. The latter discrepancy seems to be corrected by the additional constraints representing the clamping at the two ends and the vertical support of the bottom surface of the plate during the simulated welding. 


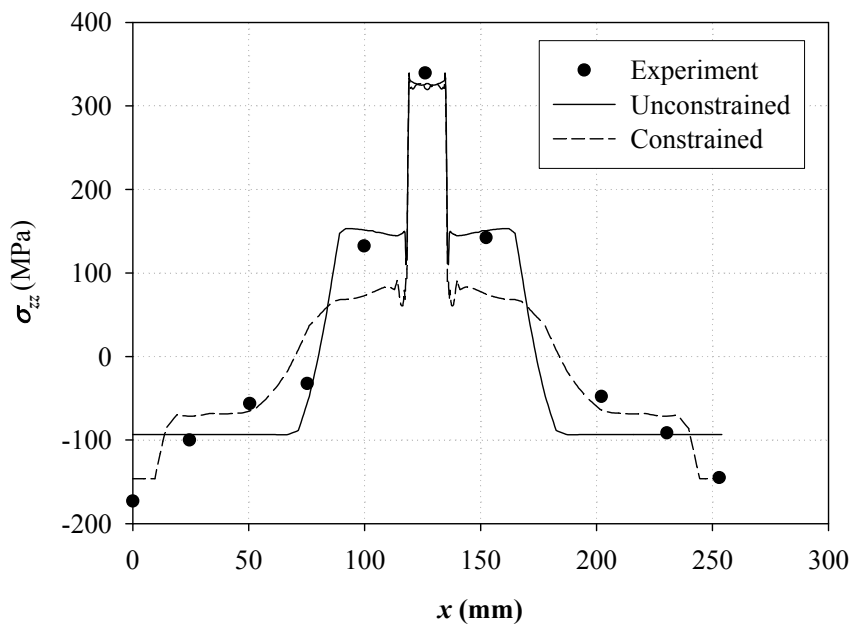

Figure 3: Longitudinal residual stress $\left(\sigma_{\mathrm{zz}}\right)$ distribution with modified plastic WM properties.

The accurate prediction of the transverse residual stress $\sigma_{x x}$ is important since it may affect the fatigue life of the joint in the presence of cracks parallel to the welding $(z)$ direction. The obtained results agreed qualitatively with other published numerical $[1,2,16]$ as well as experimental results [23]. The predicted $\sigma_{x x}$ distributions were found sensitive to variations of yield strain $\varepsilon_{Y}=\sigma_{Y} / E$, especially at high temperatures. Most of the available material data indicate that the yield strain decreases with temperature below the melting temperature. Above the melting temperature however, when the metal is still modelled as solid for numerical purposes, the plastic strain must be given a sufficiently high value to prevent spurious plastic strain developing within melted regions. Numerical experiments with increasing yield strains at temperatures above 1000 $\mathrm{K}$ did indicate a redistribution of plastic strains in and around the weld area.

\section{Fillet weld}

Fillet welding for the manufacture of $\mathrm{T}$-joints has been simulated by either considering single-fillet joints or assuming simultaneous welding on the two sides. The main objective of this analysis was to demonstrate the simulation of a sequential T-joint welding process. The element death and rebirth features of ANSYS allowed the representation of the second fillet weld during the first welding pass.

The geometry of the manufactured joint is shown in fig. 4. Thermocouples were spot-welded to the plate surface to measure the temperature development during the welding and cooling process. The complete elasto-plastic stress-strain relations for both $\mathrm{BM}$ and $\mathrm{WM}$ at $T_{r}$ were obtained experimentally. Because of the small area of the heat affected zone (HAZ), its plastic properties were deduced indirectly from micro-hardness test data. 


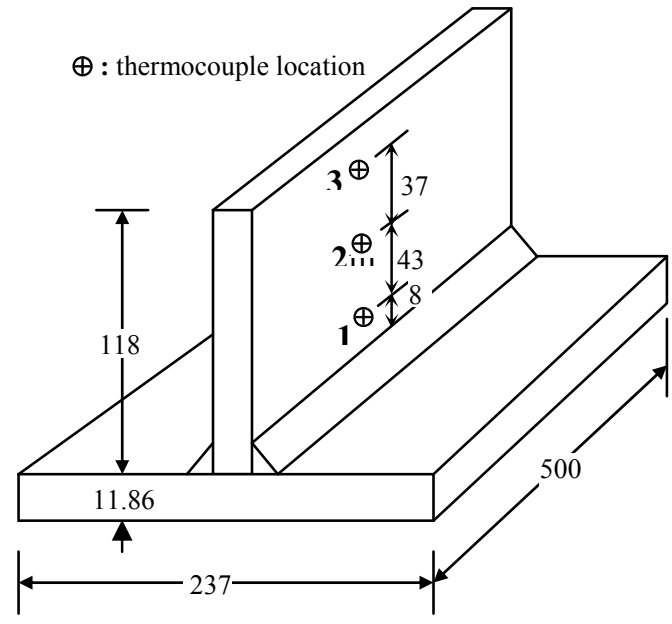

Figure 4: $\quad$ Manufactured and analysed welded T-joint (all dimensions in $\mathrm{mm}$ ).

The sizes and shapes of the two fillet welds were assumed to be the same in the FE model although they were slightly different in the specimen. Additionally, no overfill was included in the FE model. The heat transfer between the surfaces of the attachment plate and the base plate, i.e. through the fit-up, was modelled using 2-D conduction bar elements. These were changed to 2-D node-to-node structural contact elements, which are only capable of supporting compression in the direction normal to the surfaces and shear in the tangential direction.

The adopted temperature-dependent thermal properties were mainly those reported by Brown and Song [7]. The heat rate was found approximately equal to $3.9 \mathrm{~kW}$, which is equivalent to $Q=19.34 \mathrm{~kJ} / \mathrm{cm}$ for the applied welding speed of approximately $2 \mathrm{~mm} / \mathrm{s}$. The value $Q_{s} / Q_{b}=0.175$ was found to give temperature distributions that best fitted the fusion zone measured from the experiment. The surface heat flux was assumed to have a Gaussian distribution.

The value of $h(T)$ on the bottom surface of the specimen was adjusted to account for the heat loss through conduction and convection due to the specimen being welded on top of a $35 \mathrm{~mm}$ thick steel plate. Moreover, the specimen is distorted after the first pass on the right side so that the left side of the specimen will not be in contact with the supporting plate and less heat will be conducted through it. After the second pass, the specimen tends to deform in the opposite direction so that, this time, part of the bottom surface on the right side of the specimen will leave the supporting plate. Thus the convection coefficient was varied during different stages of the welding process to account for these changes in actual boundary conditions. The ramped heat input function comprised four load steps for each pass as shown in fig. 1 , where $t_{1}=t_{3}=1 \mathrm{~s}, t_{2}=5 \mathrm{~s}$, and the time between the two passes $t_{4}=677 \mathrm{~s}$.

The temperature histories at the thermocouple locations (fig. 4), obtained from both the experiment and the FE analysis, are shown in fig. 5. A good agreement between them has been achieved through the calibration of heat input and boundary convection, for which uncertainties arise regarding both their 


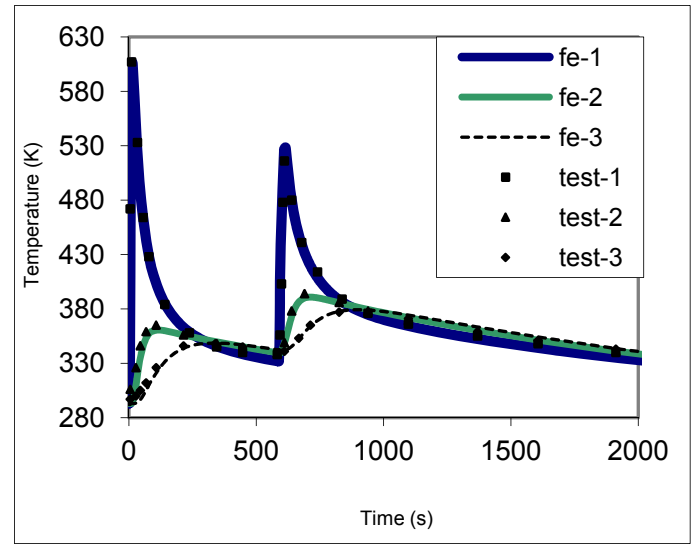

Figure 5: $\quad$ Measured and predicted temperature histories.

magnitude and distribution. The fusion zone was predicted within the limits observed in the test specimen.

Compressive stresses were observed in the heated areas counterbalanced by tensile ones away from them. This pattern was gradually reversed during cooling. The final angular distortion of the test piece was measured to be $3.4^{\circ}$, which was comparable with the respective FE prediction.

The residual $\sigma_{z z}$ distribution along the surfaces of the plate and attachment was found to be in qualitative agreement with patterns determined experimentally [20]. The simulation of sequential welding had a strong effect on results; the predicted $\sigma_{x x}$ distributions from the first and the second pass fillet were very different from those obtained from a symmetric model, representing simultaneous fillet welding on both sides of the T-joint.

\section{Conclusions}

The results of the presented FE simulations of welding processes confirmed that variations in the process parameters have little influence on the magnitude of residual stresses. They are also consistent with numerical studies, which argued that residual stress solutions are not sensitive to the thermal analysis input even if the latter has significant influence on temperature distribution [18]. Thus, an arc efficiency value chosen within the range given in the literature for a specified welding type is sufficient to give acceptably accurate residual stress results. Similarly, data on temperature-dependent thermal properties for structural steel obtained from the literature can be considered reliable. The same argument applies to the selection of data for radiation and convection parameters as well as the thermal expansion coefficient.

The residual stresses are strongly affected by $\sigma_{Y}$ and $\sigma_{u} ; \varepsilon_{Y}$ at high temperatures was also found to affect the transverse residual stress and distortion results. Modelling external constraints was particularly influential on both thermal and stress analyses results. Contact, in particular, increases considerably 
the heat loss through an interface and it has a particularly strong direct effect on the transverse residual stress and distortion predictions.

Input parameters should be chosen carefully and confidence in them gained by examining the sensitivity of residual stress results to their variation. This is essential for overcoming uncertainties in material properties, particularly at high temperatures. The assessment of an FE model based on comparison with reported experimental measurements should also take into account the uncertainties arising from unavoidable errors and limitations present in these data.

Other material-related, potentially influential factors, such as transformation plasticity, temperature-history dependency, plasma pressure, the fluid movement in the weld pool and the variation of material properties within the HAZ deserve further consideration in the context of advanced FE modelling. It is, however, important for an FE analysis to be not only reliable but also efficient by accounting only for the most influential phenomena.

\section{References}

[1] Tsai, C.L., Lee, S.G. and Shim, Y.L., Modelling techniques for weldinginduced residual stress predictions. Proceedings of the International Conference on Modelling and Control of Joining Processes, ed. T. Zacharia, AWS: Fla., pp. 462-469, 1993.

[2] Hong, J.K., Tsai, C.L. and Dong, P., Assessment of numerical procedures for residual stress analysis of multipass welds. Welding Journal Research Supplement, 77(9), pp. 372s-382s, 1998.

[3] Brown, S.B. and Song, H., Rezoning and Dynamic Substructuring Techniques in FEM Simulations of Welding Processes. Journal of Engineering for Industry-Transactions of the ASME, 115, pp. 415-423, 1993.

[4] Jonsson, M., Karlsson, L. and Lindgren, L.E., Deformations and Stresses in Butt-Welding of Large Plates with Special Reference to the Mechanical Material Properties. Journal of Engineering Materials and TechnologyTransactions of the Asme, 107(4), pp. 265-270, 1985.

[5] Wikander, L., Karlsson, L., Nasstrom, M. and Webster, P., Finite-Element Simulation and Measurement of Welding Residual- Stresses. Modelling and Simulation in Materials Science and Engineering, 2(4), pp. 845-864, 1994.

[6] Shim, Y., Feng, Z., Lee, S., Kim, D., Jaeger, J., Papritan, J.C. and Tsai, C.L., Determination of Residual-Stresses in Thick-Section Weldments. Welding Journal Research Supplement, 71(9), pp. 305s-312s, 1992.

[7] Brown, S. and Song, H., Finite element simulation of welding of large structures. Journal of Engineering for Industry-Transactions of the ASME, 114, pp. 441-451, 1992.

[8] Brown, S.B. and Song, H., Implications of 3-Dimensional Numerical Simulations of Welding of Large Structures. Welding Journal Research Supplement, 71(2), pp. 55s-62s, 1992. 
[9] Wu, A., Syngellakis, S. and Mellor, B.G., Effect of thermal input on finite element predictions of welding residual stresses. Advanced Materials Research, 399-401, pp. 1976-1983, 2011.

[10] Wu, A., Syngellakis, S. and Mellor, B.G., Finite element prediction of residual stresses in a fillet welded T-joint. Proc. of the 6th Int. Conf. on Trends in Welding Research, ed. S.A. David, T. DebRoy, J.C. Lippold, H.B. Smartt and J.M. Vitek, ASM International: Pine Mountain, GA, pp. 832-836, 2003.

[11] Argyris, J.H., Szimmat, J. and Willam, K., Computational Aspects of Welding Stress-Analysis. Computer Methods in Applied Mechanics and Engineering, 33(1-3), pp. 635-665, 1982.

[12] Bonifaz, E.A., Finite element analysis of heat flow in single-pass arc welds. Welding Journal Research Supplement, 79(5), pp. 121s-125s, 2000.

[13] Karlsson, R.I. and Josefson, B.L., Three-dimensional finite element analysis of temperatures and stresses in a single-pass butt-welded pipe. Journal of Pressure Vessel Technology-Transactions of the ASME, 112, pp. 76-84, 1990.

[14] Michaleris, P. and DeBiccari, A., Prediction of welding distortion. Welding Journal Research Supplement, 76(4), pp. 172s-181s, 1997.

[15] Lindgren, L.E., Runnemalm, H. and Nasstrom, M.O., Simulation of multipass welding of a thick plate. International Journal for Numerical Methods in Engineering, 44(9), pp. 1301-1316, 1999.

[16] Andersson, B.A.B., Thermal stresses in a submerged-arc welded joint considering phase transformations. Journal of Engineering Materials and Technology-Transactions of the ASME, 100, pp. 356-352, 1978.

[17] Ueda, Y. and Yuan, M.G., Prediction of Residual-Stresses in Butt Welded Plates Using Inherent Strains. Journal of Engineering Materials and Technology-Transactions of the Asme, 115(4), pp. 417-423, 1993.

[18] Song, J., Peters, J., Noor, A. and Michaleris, P., Sensitivity analysis of the thermomechanical response of welded joints. International Journal of Solids and Structures, 40(16), pp. 4167-4180, 2003.

[19] Touloukian, Y.S., Thermophysical properties of high temperature solid materials, Volume 3: Ferrous alloys, Macmillan: New York, pp. 1967.

[20] Rao, N.R.N. and Tall, L., Residual stresses in welded plates. Welding Journal Research Supplement, 40(10), pp. 468s-480s, 1961.

[21] Masubuchi, K., Analysis of welded structures, Pergamon Press: Cambridge, MA, pp. 1980.

[22] Christensen, N., Davies, V.L. and Gjermundsen, K., Distribution of temperatures in arc welding. British Welding Journal, 12(2), pp. 54-75, 1965.

[23] Dilthey, U., Reisgen, U. and Kretschmer, M., Comparison of FEM simulations to measurements of residual stresses for the example of a welded plate: a state-of-the-art report. Modelling and Simulation in Materials Science and Engineering, 8(6), pp. 911-926, 2000. 